\title{
Jet quenching parameter with hyperscaling violation
}

\author{
J. Sadeghi ${ }^{\mathrm{a}}$, S. Heshmatian ${ }^{\mathrm{b}}$ \\ Department of Nuclear Physics, Faculty of Basic Sciences, University of Mazandaran, P. O. Box 47415-416, Babolsar, Iran
}

Received: 25 October 2013 / Accepted: 31 March 2014 / Published online: 4 September 2014

(C) The Author(s) 2014. This article is published with open access at Springerlink.com

\begin{abstract}
In this paper we study the behavior of the jet quenching parameter in a background metric with hyperscaling violation at finite temperature. The background metric is covariant under a generalized Lifshitz scaling symmetry with the dynamical exponent $z$ and hyperscaling exponent $\theta$. We have evaluated the jet quenching parameter for a certain range of these parameters which are consistent with the Gubser bound conditions in terms of $T, z$, and $\theta$. The results are compared with those of experimental data as well as conformal and the non-conformal cases. Finally, we add a constant electric field to the background and find its effect on the jet quenching parameter.
\end{abstract}

\section{Introduction}

Holography is a powerful tool to map a $D$ dimensional strongly coupled field theory at large $N$ limit to a $D+1$ dimensional gravitational theory at weak coupling [1-4]. The gauge/gravity duality has been used to study the QCD and hadron physics. The dynamics of a moving quark and the motion of a quark-antiquark pair in a strongly coupled plasma have been studied in the context of gauge/gravity duality [5-29]. Also, the computations of the QCD parameters demonstrate the efficiency of this duality [20-29].

As a result of broad application of this characteristic, generalizations of metrics dual to field theories have been proposed. One of such generalizations is to use metrics dual to field theories which are not scale invariant, while they are conformal to Lifshitz spacetimes [30-36]. These backgrounds have a dynamical Lifshitz parameter $z$ as well as a hyperscaling violation exponent $\theta$. Such metrics have been used to describe condensed matter systems and string theory solutions [37-57]. Lorentz symmetry represents a foundation

\footnotetext{
a e-mail: j.sadeghi@umz.ac.ir

be-mail: s.heshmatian@umz.ac.ir
}

of both general relativity and the standard model. Therefore, Lorentz invariance violation may lead to new physics.

Due to the quark confinement, when the quark and antiquark are moving apart from each other, they will be hadronized by creating more pairs as jets. Some of the jets are suppressed by the medium and lose energy according to the jet quenching phenomenon. The jet quenching parameter is the probability of the jet quenching. It is proportional to the momentum fluctuation as $\hat{q} \propto\left\langle\vec{p}_{\perp}^{2}\right\rangle$ [58-64]. However, in a dual description, this parameter is related to the coefficient in the exponent of an adjoint Wilson loop [65-79].

In this paper, we use the gauge/gravity duality to study the behavior of the jet quenching parameter in the background metric with hyperscaling violation at finite temperature with and without a constant B-field. This paper is organized as follows: in Sect. 2, we briefly review the background of Ref. [56]. The metric is covariant under a generalized Lifshitz scaling symmetry and has a generic Lorentz violating form. In Sect. 3, the holographic description of strongly coupled QFT has been applied to illustrate the behavior of the jet quenching parameter at finite temperature. We determine the allowed regions for $z$ and $\theta$ and evaluate the jet quenching parameter numerically in terms of the background Hawking temperature, $z$, and $\theta$ parameters. In Sect. 4, we add a constant B-field (electric field) to the background metric to estimate how the jet quenching parameter depends on the Hawking temperature and also the electric field.

\section{Review of the background}

In this section, we briefly review the background introduced in Ref. [56]. The authors have considered a background metric in which Lorentz invariance is broken. It has been argued that although charge densities induce a trivial (gapped) behavior at low energy/temperature, in special cases there are non-trivial IR fixed points (quantum critical points) in which the theory is scale invariant and has a 
generic Lorentz violating form. A generalized Lifshitz metric is introduced in Refs. [39,40,48-50] and has the following form:

$d s^{2}=u^{\theta}\left[-\frac{d t^{2}}{u^{2 z}}+\frac{b_{0} d u^{2}+d x^{i} d x^{i}}{u^{2}}\right]$,

where $b_{0}=\ell^{2}$ and $\ell$ is the IR scale. This metric is covariant under a generalized Lifshitz scaling symmetry,

$t \rightarrow \lambda^{z} t, u \rightarrow \lambda u, x^{i} \rightarrow \lambda x^{i}, d s^{2} \rightarrow \lambda^{-\theta} d s^{2}$.

The exponent $z$ is the Lifshitz parameter and the exponent $\theta$ is the hyperscaling violation exponent, which is responsible for the non-standard scaling of physical quantities and controls the transformation of the metric. The scalar curvature corresponding to these geometries is given by

$R=-\frac{3 \theta^{2}-4(z+3) \theta+2\left(z^{2}+3 z+6\right)}{b_{0}} u^{-\theta}$.

The geometries are flat for $\theta=2$ and $z=0,1$. The $(\theta=0, z=1)$ geometry is in Rindler coordinates and it is Ricci flat when $\theta=4$ and $z=3$. These special solutions violate the conditions of Ref. [56]. For $\theta=0$, the scalar curvature is constant (pure Lifshitz case, Ref. [36]).

Using the following radial redefinition:

$u=(2-z) r^{\frac{1}{2-z}}$

and rescaling $t$ and $x^{i}$, the following metric is obtained:

$d s^{2} \sim r^{\frac{\theta-2}{2-z}}\left[-f(r) d t^{2}+\frac{d r^{2}}{f(r)}+d x^{i} d x^{i}\right]$,

$f(r)=f_{0} r^{2 \frac{1-z}{2-z}}$

where $f_{0}=(2-z)^{2(1-z)}$. The energy scale is given by the scaling of the $g_{t t}$ component of the metric. So, in the presence of hyperscaling violations, one obtains

$E \simeq u^{\frac{\theta-2 z}{2}} \simeq r^{\frac{\theta-2 z}{2(2-z)}}$

The Gubser criterion is a necessary condition for the acceptability of a naked singularity [80]. For the generalized scaling solutions of Eq. (5) the Gubser bound conditions become $[39,40,56]$

$$
\begin{aligned}
& \frac{2 z+3(2-\theta)}{2(z-1)-\theta}>0, \quad \frac{z-1}{2(z-1)-\theta}>0, \\
& \frac{2(z-1)+3(2-\theta)}{2(z-1)-\theta}>0,
\end{aligned}
$$

and the thermodynamic stability implies that

$\frac{z}{2(z-1)-\theta}>0$

Equations (7) and (8) lead to $z(z-1)>0$. The authors of Ref. [56] have also considered several cases for the generalized Lifshitz geometries:

- 1a) $\frac{\theta-2}{z-2}>0$ and $\frac{z-1}{z-2}>0$.

- 1b) $\frac{\theta-2}{z-2}>0$ and $\frac{z-1}{z-2}<0$.

- 2a) $\frac{\theta-2}{z-2}<0$ and $\frac{z-1}{z-2}>0$.

- 2b) $\frac{\theta-2}{z-2}<0$ and $\frac{z-1}{z-2}<0$.

In the first two cases, the boundary is at $r=0$ and in the latter two cases, the boundary is at $r=\infty$. For the last case, there are no acceptable geometries surviving the Gubser bounds in Eq. (7).

The generalization of the metrics in Eq. (5) to include finite temperature is

$$
\begin{gathered}
d s^{2} \sim\left(\frac{r}{\ell}\right)^{-\alpha}\left[-f(r) d t^{2}+\frac{d r^{2}}{f(r)}+d x^{i} d x^{i}\right], \\
f(r)=f_{0}\left(\frac{r}{\ell}\right)^{2 \beta} h \quad, \quad h=1-\left(\frac{r}{r_{h}}\right)^{\gamma},
\end{gathered}
$$

where $\ell$ is the IR scale, $\alpha=\frac{\theta-2}{z-2}, \beta=\frac{z-1}{z-2}$, and $\gamma=$ $\frac{z+\frac{3}{2}(2-\theta)}{2-z}$.

\section{Jet quenching parameter}

In this section we analyze the behavior of the jet quenching parameter for the background metric of Eq. (9). In the holographic description, the jet quenching parameter is related to the Wilson loop joining two light-like lines by the following equation [69-71]:

$\left\langle\mathcal{W}^{A}(C)\right\rangle \simeq \exp \left(-\frac{1}{4 \sqrt{2}} \hat{q} L^{-} L^{2}\right)$

where $C$ is the null-like rectangular Wilson loop formed by a dipole with heavy $q \bar{q}$ pair. The quark and antiquark are separated by a small length $L$ and travel along the $L^{-}$direction. By applying the relations $\left\langle\mathcal{W}^{F}(C)\right\rangle^{2} \simeq\left\langle\mathcal{W}^{A}(C)\right\rangle$ and $\left\langle\mathcal{W}^{F}(C)\right\rangle \simeq e^{-S_{I}}$, the jet quenching parameter is given by [69-71]

$\hat{q} \equiv 8 \sqrt{2} \frac{S_{I}}{L^{-} L^{2}}$, 
where $S_{I}=S-S_{0}$. Here $S$ is the Nambu-Goto action of the fundamental string, $S_{0}$ is the self-energy from the mass of two quarks and $S_{I}$ is the regularized string worldsheet action.

To evaluate the jet quenching parameter we start with the background of Eq. (9), and use the following light-cone coordinates:

$x^{ \pm}=\frac{t \pm x^{1}}{\sqrt{2}}$,

to rewrite the black hole metric as

$$
\begin{aligned}
d s^{2}= & b^{2}(r)\left[-(1+f(r)) d x^{+} d x^{-}+\frac{1-f(r)}{2}\left[\left(d x^{+}\right)^{2}\right.\right. \\
& \left.\left.+\left(d x^{-}\right)^{2}\right]+\left(d x^{2}\right)^{2}+\left(d x^{3}\right)^{2}+f^{-1}(r) d r^{2}\right],
\end{aligned}
$$

where $b^{2}(r)=(r / \ell)^{-\alpha}$. We choose the static gauge $\tau=$ $x^{-}\left(0 \leq x^{-} \leq L^{-}\right), \sigma=x^{2}\left(-\frac{L}{2} \leq x_{2} \leq \frac{L}{2}\right)$, and we consider the string with endpoints located at $\sigma= \pm \frac{L}{2}$. In the limit $L^{-} \gg L$, the $x^{-}$dependence of the worldsheet can be neglected and the string profile is completely specified by $r=r(\sigma)$.

The induced metric of the fundamental string can be calculated as

$g_{\alpha \beta}=b^{2}(r)\left(\begin{array}{cc}-\frac{f(r)-1}{2} & 0 \\ 0 & 1+\frac{r^{\prime 2}}{f(r)}\end{array}\right)$.

Plugging the above equation into the Nambu-Goto action, we obtain

$$
\begin{aligned}
S= & -\frac{1}{2 \pi \alpha^{\prime}} \int d \tau d \sigma \sqrt{-\operatorname{det} g_{\alpha \beta}}=\frac{L^{-}}{\sqrt{2} \pi \alpha^{\prime}} \int_{0}^{\frac{L}{2}} \\
& \times d y b^{2}(r) \sqrt{(f(r)-1)\left(1+\frac{r^{\prime 2}}{f(r)}\right) .}
\end{aligned}
$$

Since the Lagrangian density does not explicitly depend on $y$, the corresponding Hamiltonian is conserved. So we have

$\frac{\partial \mathcal{L}}{\partial r^{\prime}} r^{\prime}-\mathcal{L}=E$,

where $r^{\prime}=d r / d y$. Here, $E$ is the constant energy of motion and $\mathcal{L}$ is the integrand of Eq. 15. Then we obtain the equation of motion for $r$ as

$r^{\prime}=\sqrt{f(r)\left[\frac{b^{4}(r)(f(r)-1)}{2 E^{2}}-1\right]}$.

Since for the black hole solution, we have $f(r)=0$ at the horizon and we are interested in the small $E$ case, the factor under the square root is always positive near the boundary and negative near the black hole horizon. Therefore, the turning point $r_{\min }\left(>r_{h}\right)$ can be obtained by solving the following equation:

$f\left(r_{\min }\right)-1=0$.

Substituting Eq. (17) in the Nambu-Goto action of Eq. (15), we have

$$
\begin{aligned}
S= & \frac{L^{-}}{\sqrt{2} \pi \alpha^{\prime}} \int_{r_{\min }}^{r_{b}} d r b^{2}(r) \sqrt{\frac{f(r)-1}{f(r)}} \\
& \times\left[1-\frac{2 E^{2}}{b^{4}(r)(f(r)-1)}\right]^{-\frac{1}{2}},
\end{aligned}
$$

where $r_{b}$ is the location of the boundary. Also we have used the relation $r^{\prime}=d r / d y$. In the small $E$ limit, we expand Eq. (19) up to the leading order and rewrite the string action as

$$
\begin{aligned}
S \simeq & \frac{L^{-}}{\sqrt{2} \pi \alpha^{\prime}} \int_{r_{\min }}^{r_{b}} d r b^{2}(r) \sqrt{\frac{f(r)-1}{f(r)}} \\
& \times\left[1+\frac{2 E^{2}}{b^{4}(r)(f(r)-1)}\right] .
\end{aligned}
$$

This action is divergent and to eliminate the divergence, it should be subtracted by the inertial mass of two free quarks, which is given by

$S_{0}=\frac{L^{-}}{\sqrt{2} \pi \alpha^{\prime}} \int_{r_{\min }}^{r_{b}} d r b^{2}(r) \sqrt{\frac{f(r)-1}{f(r)}}$.

Here, we have used the gauge $x^{-}=\tau$ and $r=\sigma$. On the other hand, the distance between the two quarks is obtained by integrating of Eq. (17):

$$
\begin{aligned}
\frac{L}{2}= & \int_{r_{\min }}^{r_{b}} d r \frac{\sqrt{2} E}{\sqrt{b^{4}(r) f(r)(f(r)-1)}} \\
& \times\left[1-\frac{2 E^{2}}{b^{4}(r)(f(r)-1)}\right]^{-\frac{1}{2}} .
\end{aligned}
$$

Again by expanding this equation in terms of $E$ and considering the small $L$ limit, we obtain the following relation:

$$
\frac{L}{2 E} \simeq \int_{r_{\min }}^{r_{b}} d r \sqrt{\frac{2}{b^{4}(r) f(r)(f(r)-1)}} .
$$

Also we can expand $E$ up to the leading order of $L$ to obtain

$$
E \simeq \frac{L}{\sqrt{2} \int_{r_{\min }}^{r_{b}} \frac{d r}{b^{4}(r) f(r)(f(r)-1)}} .
$$

The regularized string worldsheet action is then given by

$$
S_{I}=S-S_{0}=\frac{L^{-} L^{2}}{8 \sqrt{2} \pi \alpha^{\prime}} \frac{1}{\int_{r_{\min }}^{r_{b}} \frac{d r}{b^{2}(r) \sqrt{f(r)(f(r)-1)}}},
$$


where we have used Eqs. (23) and (24). Finally, the jet quenching parameter for the scaling metric with hyperscaling violation is acquired as follows:

$\hat{q}_{\mathrm{LV}}=\frac{1}{\pi \alpha^{\prime}}\left(\int_{r_{\min }}^{r_{b}} \frac{d r}{b^{2}(r) \sqrt{f(r)(f(r)-1)}}\right)^{-1}$.

In the conformal limit $(\theta=0$ and $z=1)$ this equation reduces to

$\hat{q}_{\text {conf. }}=\sqrt{2 \lambda} \pi^{\frac{3}{2}} T^{3} \frac{\Gamma\left[\frac{3}{4}\right]}{\Gamma\left[\frac{5}{4}\right]}$,

which gives $\hat{q}_{\text {conf. }}=1.95 \mathrm{GeV}^{2} / \mathrm{fm}$ for $T=T_{c}$ and $\lambda=5.5$. The Hawking temperature associated to the black hole metric (9) is given by

$T=\frac{1}{4 \pi}\left|\frac{g_{00}^{\prime}}{\sqrt{-g_{00} g_{11}}}\right|_{r=r_{h}}=\frac{f_{0}}{8 \pi l}\left(\frac{r_{h}}{l}\right)^{\frac{z}{z-2}}\left|\frac{2 z-3 \theta+6}{z-2}\right|$.

In order to evaluate the jet quenching parameter of Eq. (26) we should first determine the allowed region for $z$ and $\theta$ parameters. In this paper, we consider the space boundary at $r=\infty$, consequently the $\alpha$ parameter is negative. In this case, to have an acceptable geometry satisfying the Gubser conditions of Eq. (7), the $\beta$ parameter should be positive.

Also, to avoid divergence of the integrand in Eq. (26) at $r \rightarrow \infty$ as well as to have a well-defined behavior for the jet quenching parameter, we choose $\frac{5}{6} \leq \beta<1$ and $-3 \leq$ $\gamma \leq-2$. These constraints together imply that the allowed geometries have the following $z$ and $\theta$ range:

$-\frac{3}{2} \theta+7 \leq z \leq-4$ for $\frac{22}{3} \leq \theta \leq \frac{34}{3}$,

$-\frac{3}{2} \theta+7 \leq z \leq-\frac{3}{4} \theta+\frac{9}{2}$

for $\theta>\frac{34}{3}$.

The allowed geometries with the above constraints are shown in the $(z, \theta)$ plane in Fig. 1.

To compare our results with those of the conformal case and experimental data and study the behavior of the jet quenching parameter $\hat{q}_{\mathrm{LV}}$, we plot it numerically for the $z=-8$ and $\theta=10$ case and we have $q_{\text {conf. }}$ as a function of temperature in Fig. 2.

From Fig. 2, one can see that at $T_{c} \simeq 250 \mathrm{MeV}, \hat{q}_{\mathrm{LV}} \simeq$ $3.56 \mathrm{GeV}^{2} / \mathrm{fm}$, which is larger than $\hat{q}_{\text {conf. }}$. As the temperature increases, $\hat{q}_{\mathrm{LV}}$ approaches $\hat{q}_{\text {conf. }}$ and $T \simeq 460 \mathrm{MeV}$, and they get equal values. Eventually, for $T>460 \mathrm{MeV}$, the values of $\hat{q}_{\mathrm{LV}}$ become smaller than $\hat{q}_{\text {conf. }}$.

In Fig. 3 we demonstrate $\hat{q}_{\mathrm{LV}} / \hat{q}_{\text {conf. }}$ as a function of temperature for the $z=-8$ and $\theta=10$ case. The numerical values of $\hat{q}_{\mathrm{LV}}$ for different temperatures are shown in Table 1 .

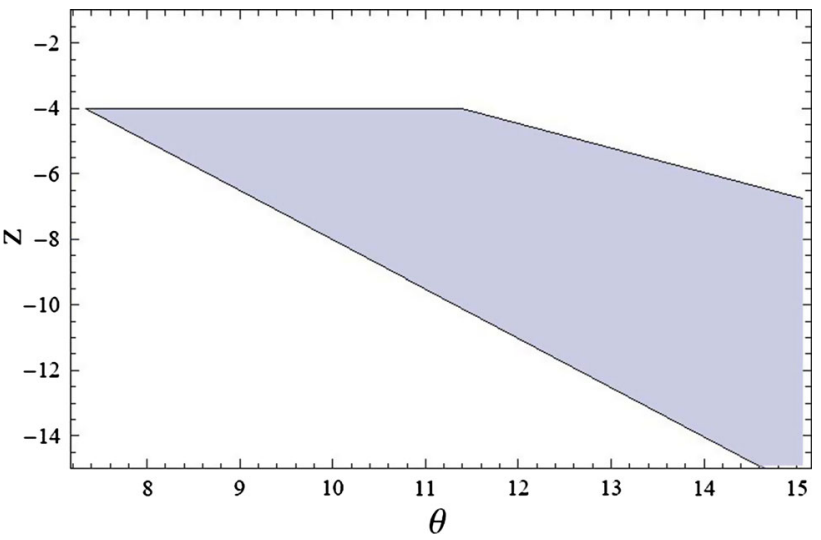

Fig. 1 Generalized Lifshitz geometries in the $(z, \theta)$ plane with $\alpha<0$, $\frac{5}{6} \leq \beta<1$, and $-3 \leq \gamma \leq-2$

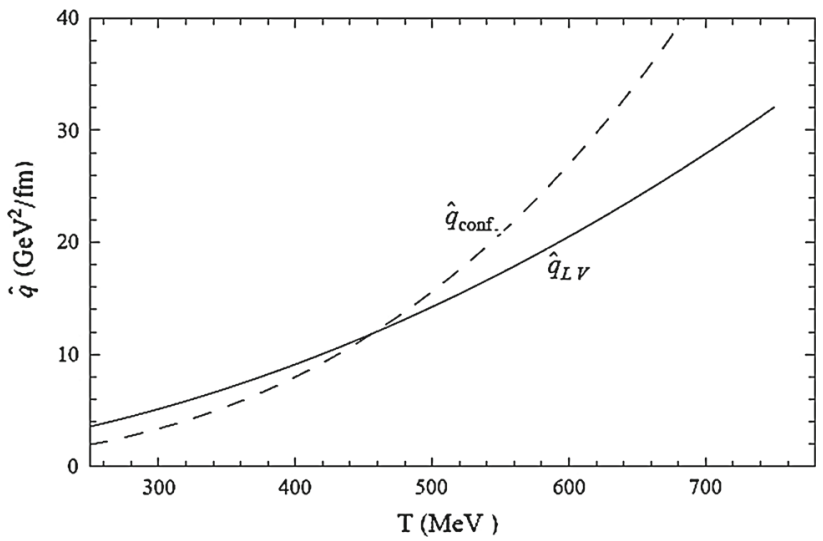

Fig. 2 Jet quenching parameter as a function of T for the scaling metric with hyperscaling violation (solid line) and the conformal case (dashed line) for $z=-8$ and $\theta=10$ case

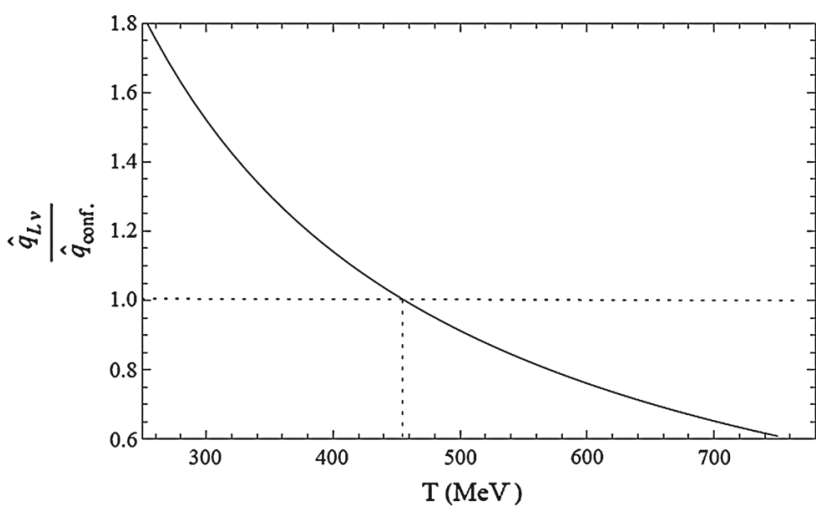

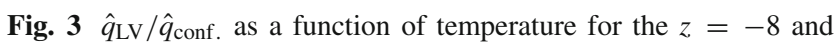
$\theta=10$ case. For $T<T_{0}$ the ratio is larger than 1 ; for $T>T_{0}$, the ratio is less than 1

From the numerical values of the jet quenching parameter, it could be realized that in the range of $300<T<500$, $5.1<\hat{q}_{\mathrm{LV}}<14.2$, which is consistent with those obtained from RHIC with $\overline{\hat{q}} \simeq 5-15 \mathrm{GeV}^{2} / \mathrm{fm}[81,82]$. 
Table 1 Jet quenching parameter $\hat{q}_{\mathrm{LV}}$ in units of $\mathrm{GeV}^{2} / \mathrm{fm}$ for different values of temperature. The temperature is given in units of the critical temperature $T_{c}$

\begin{tabular}{llllll}
\hline & $T=T_{c}$ & $T=1.5 T_{c}$ & $T=2 T_{c}$ & $T=2.5 T_{c}$ & $T=3 T_{c}$ \\
\hline$\hat{q}_{\mathrm{LV}}$ & 3.56 & 8.01 & 14.24 & 22.25 & 32.05 \\
\hline
\end{tabular}

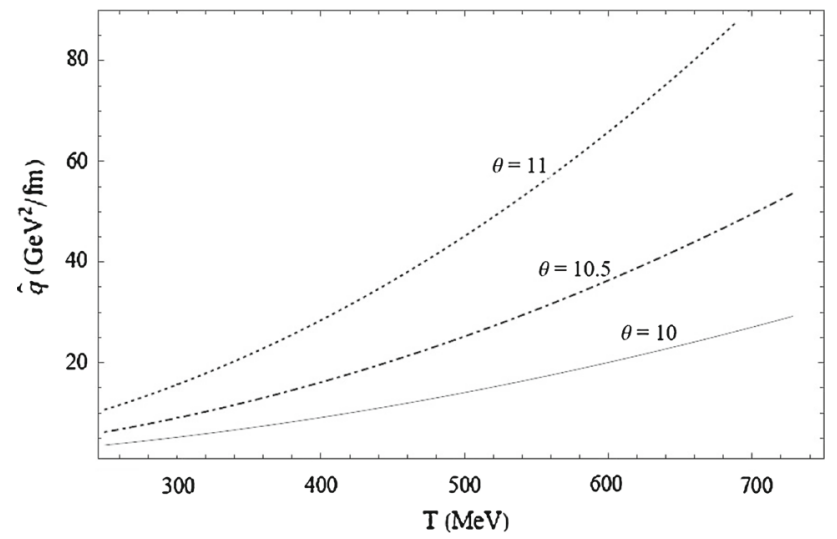

Fig. $4 \hat{q}_{\mathrm{LV}}$ as a function of temperature for different values of $\theta$ and constant value of the Lifshitz exponent $z=-8$. As $\theta$ increases, the jet quenching parameter increases

Now, we assume two different cases to examine the behavior of the jet quenching parameter $\hat{q}_{\mathrm{LV}}$ as a function of $T$ for different values of $z$ and $\theta$ parameters.

- To evaluate the jet quenching parameter as a function of temperature for different values of $\theta$ and fixed Lifshitz exponent $(z=-8)$. This case is shown in Fig. 4. From this plot, one can observe that $\hat{q}_{\mathrm{LV}}$ rises as $\theta$ increases.

- To evaluate the jet quenching parameter as a function of temperature for different values of $z$ and fixed hyperscaling exponent $(\theta=10)$. This case is shown in Fig. 5. Similar to the previous case, $\hat{q}_{\mathrm{LV}}$ is an increasing function of $z$.

Then we plot the jet quenching parameter as a function of the hyperscaling exponent $\theta$ for different values of $T$ and fixed Lifshitz exponent $z=-8$ in Fig. 6. From this figure, it is clear that $\hat{q}_{\mathrm{LV}}$ is an increasing function of $\theta$.

Finally $\hat{q}_{\mathrm{LV}}$ as a function of the Lifshitz exponent is depicted in Fig. 7 for different values of $T$ and fixed hyperscaling violation exponent $\theta=10$. Like the previous case, it is an increasing function of $z$.

\section{Effect of constant electric field}

In the previous section, we evaluated the jet quenching parameter in the scaling background with hyperscaling vio-

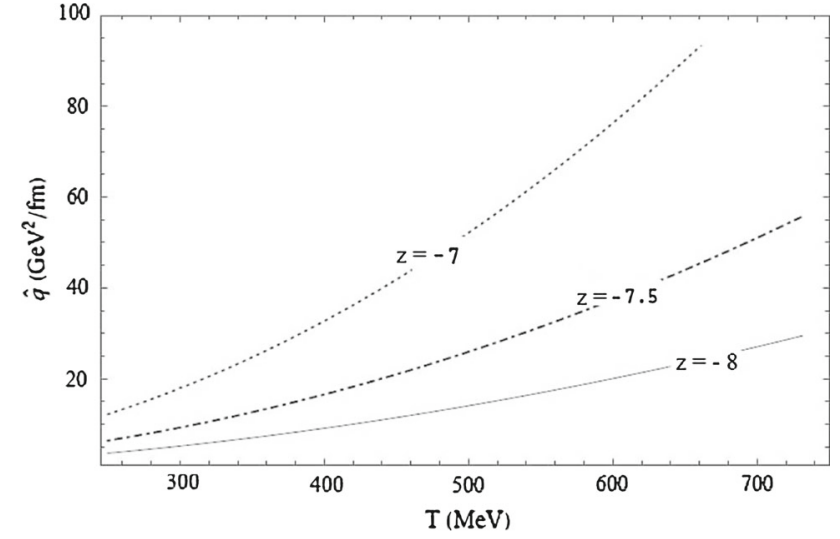

Fig. $5 \hat{q}_{\mathrm{LV}}$ as a function of temperature for different values of $z$ and fixed hyperscaling exponent $\theta=10$. The jet quenching parameter is an increasing function of $z$

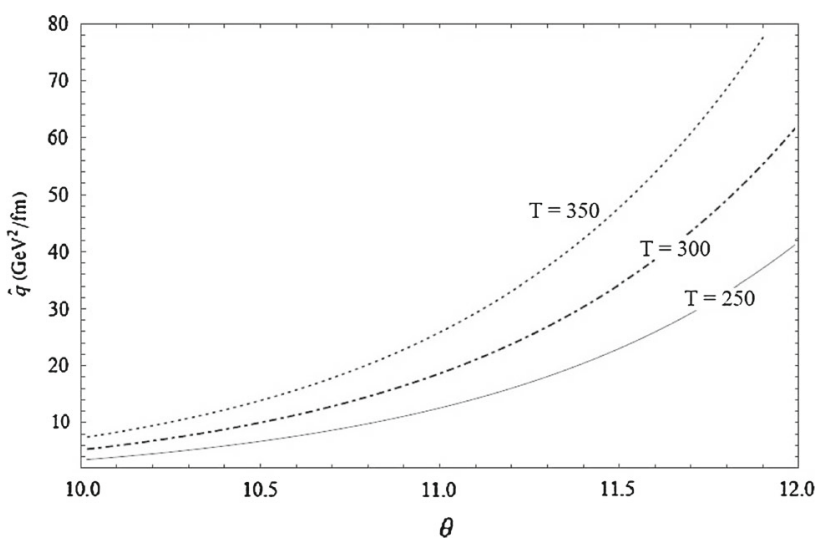

Fig. $6 \hat{q}_{\mathrm{LV}}$ as a function of $\theta$ for different values of $T$ and fixed $z=-8$. As $\theta$ increases, the jet quenching parameter increases

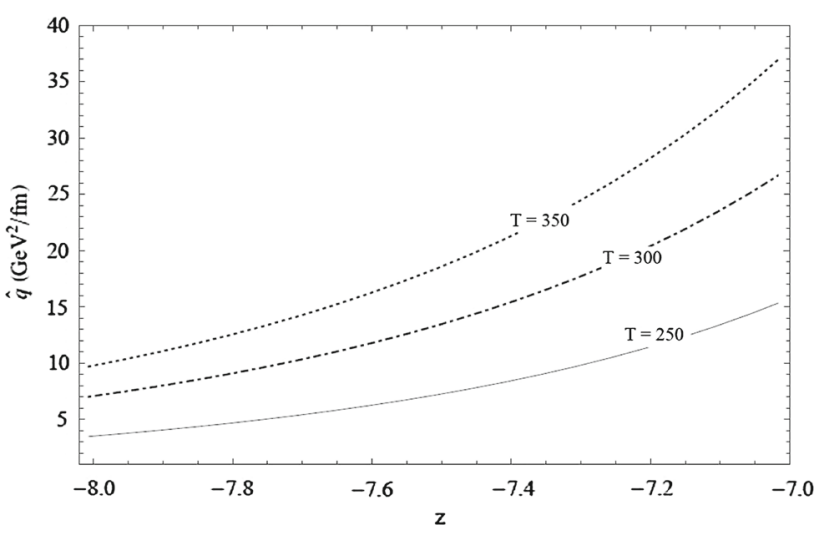

Fig. $7 \hat{q}_{\mathrm{LV}}$ as a function of $z$ for different values of $T$ and fixed $\theta=10$. As $z$ increases, the jet quenching parameter increases

lation at finite temperature introduced in Ref. [56]. Now, we study the effect of a constant electric field on the jet quenching parameter following the method proposed in Ref. [19]. In this setup, the constant B-field is considered to be along the $x^{1}$ and $x^{2}$ directions. Due to the fact that only the field strength is involved in the equations of motion, this ansatz could be 
a good solution to supergravity and this is the minimal setup for the dual field theory to study the B-field correction. The constant B-field is added to the line element of Eq. (9) by the following two-form:

$B=B_{01} d t \wedge d x_{1}+B_{12} d x_{1} \wedge d x_{2}$,

where $B_{01}=\mathcal{E}$ and $B_{12}=\mathcal{H}$ are NS-NS antisymmetric electric and magnetic fields which are assumed to be constant.

In this paper we consider $B_{12}=0$ and study the effect of a constant electric field on the evolution of the jet quenching parameter. Similar to the previous section, we use the static gauge $\tau=x^{-}, \sigma=x^{2}$ and the light-cone coordinates of Eq. (12). In the presence of an electric field, the string action is described by

$$
S=-\frac{1}{2 \pi \alpha^{\prime}} \int d \tau d \sigma \sqrt{-\operatorname{det}(g+b)_{\alpha \beta}},
$$

where $\alpha, \beta=\tau, \sigma$, and $g_{\alpha \beta}$ is given in Eq. (14). In the lightcone coordinates, the induced b-field on the string worldsheet, $b_{\alpha \beta}=B_{\mu \nu} \partial_{\alpha} X^{\mu} \partial_{\beta} X^{\nu}$, is obtained as

$b_{\alpha \beta}=\left(\begin{array}{cc}\frac{\mathcal{E}}{2} & 0 \\ 0 & 0\end{array}\right)$.

Putting Eqs. (14) and (32) into Eq. (31) for the string action leads to

$S=\frac{L^{-}}{\sqrt{2} \pi \alpha^{\prime}} \int_{0}^{\frac{L}{2}} d y b^{2}(r) \sqrt{\left(f(r)-1-\frac{\mathcal{E}}{b^{2}(r)}\right)\left(1+\frac{r^{\prime 2}}{f(r)}\right)}$.

Also, the equation of motion for $r$ is found to be

$r^{\prime 2}=f(r)\left[\frac{b^{4}(r)}{2 E^{2}}\left(f(r)-1-\frac{\mathcal{E}}{b^{2}(r)}\right)-1\right]$.

Inserting this equation into Eq. (33) and taking the $E \rightarrow 0$ limit, we obtain the following equation for the string action:

$$
\begin{aligned}
S= & \frac{L^{-}}{\sqrt{2} \pi \alpha^{\prime}} \int_{r_{\min }}^{\infty} d r b^{2}(r) \sqrt{\frac{f(r)-1-\frac{\mathcal{E}}{b^{2}(r)}}{f(r)}} \\
& \times\left[1+\frac{2 E^{2}}{b^{4}(r)\left(f(r)-1-\frac{\mathcal{E}}{b^{2}(r)}\right)}\right],
\end{aligned}
$$

and the following equation for the self-energy of two quarks:

$$
S_{0}=\frac{L^{-}}{\sqrt{2} \pi \alpha^{\prime}} \int_{r_{\min }}^{\infty} d r b^{2}(r) f^{-1}(r) \sqrt{f(r)-1-\frac{\mathcal{E}}{b^{2}(r)}} .
$$

Also the distance between two end points of string becomes

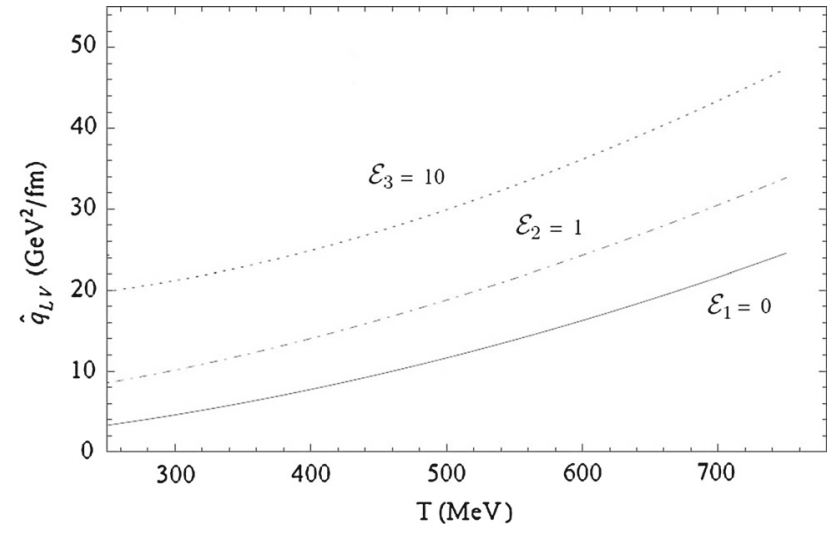

Fig. $8 \hat{q}_{\mathrm{LV}}$ as a function of $T$ for different values of $\mathcal{E}\left(\mathcal{E}_{1}<\mathcal{E}_{2}<\mathcal{E}_{3}\right)$. As $T$ increases, the jet quenching parameter increases

$L=2 \sqrt{2} E \int_{r_{\min }}^{\infty} d r\left[b^{4}(r) f(r)\left(f(r)-1-\frac{\mathcal{E}}{b^{2}(r)}\right)\right]^{-\frac{1}{2}}$.

Finally, we obtain the jet quenching parameter as

$\hat{q}_{\mathrm{LV}}=\frac{1}{\pi \alpha^{\prime}}\left(\int_{r_{\min }}^{\infty} \frac{d r}{b^{2}(r) \sqrt{f(r)\left(f(r)-1-\frac{\mathcal{E}}{b^{2}(r)}\right)}}\right)^{-1}$,

where $r_{\min }$ is obtained by solving the following equation numerically:

$f\left(r_{\min }\right)-1-\frac{\mathcal{E}}{b^{2}\left(r_{\min }\right)}=0$.

Now we proceed to evaluate the jet quenching parameter numerically and study its behavior in the presence of a constant electric field. For this purpose, we consider two different cases with numerical values $z=-8$ for the Lifshitz exponent and $\theta=10$ for the hyperscaling violation exponent.

Firstly, we numerically evaluate the jet quenching parameter as a function of temperature for different values of $\mathcal{E}$. From this plot, one can observe that $\hat{q}_{\mathrm{LV}}$ becomes large as $T$ increases. This case is shown in Fig. 8.

Secondly, we numerically evaluate the jet quenching parameter as a function of $\mathcal{E}$ for different values of $T$ in the units of $T_{c}$. From this plot we can see that at constant temperature, $\hat{q}_{\mathrm{LV}}$ rises as $T$ increases. This case is shown in Fig. 9.

\section{Results and discussion}

Jet quenching of partons produced at RHIC with high transverse momentum is one of the interesting properties of a 


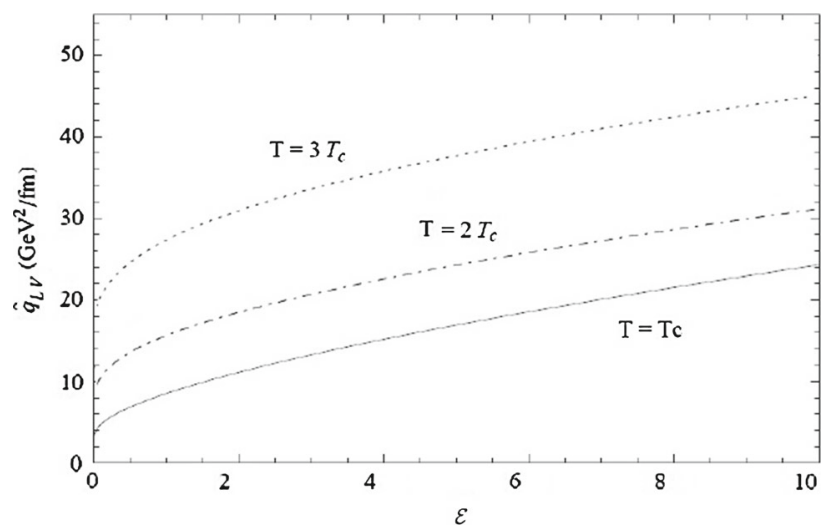

Fig. $9 \hat{q}_{\mathrm{LV}}$ as a function of $\mathcal{E}$ for different values of $T$ in units of $T_{c}$. The jet quenching parameter increases as $\mathcal{E}$ increases

strongly coupled plasma. It is possible to determine this quantity using the gauge/gravity duality for gauge theories at finite temperature.

In the non-conformal background of Ref. [29], the dependency of the jet quenching parameter on the probe quark momentum has been investigated for charm and bottom quarks for the temperature range relevant to the RHIC and the ALICE experiment at LHC. The authors demonstrated that, except at low momenta, $\widehat{q}_{\perp}$ varies in the $1-5 \mathrm{GeV}^{2} / \mathrm{fm}$ range for $T=250 \mathrm{MeV}$. Also, the authors of Ref. [66] found that $\widehat{q}$ grows with temperature, but slower than the $T^{3}$ growth of $N=4 \mathrm{SYM}$. For $T=290 \mathrm{MeV}$, which in the alternative energy scheme corresponds to $T=395 \mathrm{MeV}$ in their holographic model, they found that $\widehat{q} \simeq 2 \mathrm{GeV}^{2} / \mathrm{fm}$. In Ref. [65], the authors have proposed a backreacting supergravity solution dual to a flavored version of the $N=4 \mathrm{SYM}$ plasma, with massless flavors. It has been shown that the value of the jet quenching parameter increases as compared to the one in $N=4$ SYM and it is shifted towards the RHIC phenomenological data.

In this paper we analyzed the behavior of the jet quenching parameter in the background metric which is covariant under a generalized Lifshitz scaling symmetry and has a generic Lorentz violating form at finite temperature.

In Sect. 3, we used the holographic description to study the jet quenching parameter at finite temperature. For this purpose, we determined the appropriate range for $z$ and $\theta$ and evaluated the jet quenching parameter numerically in terms of $T, z$, and $\theta$ parameters. We considered the space boundary at $r=\infty$; consequently, the $\alpha$ parameter is negative. In this case, to have an acceptable geometry satisfying the Gubser conditions, the $\beta$ parameter should be positive. To avoid the divergence of the jet quenching integrand at $r \rightarrow \infty$ and to have a well-defined behavior for the jet quenching parameter, we chose $\frac{5}{6} \leq \beta<1$ and $-3 \leq \gamma \leq-2$. We plotted $\hat{q}_{\mathrm{LV}}-T$ numerically for $z=-8$ and $\theta=10$ as a function of temperature in Fig. 2, which is qualitatively similar to Refs. [25] and [29]. At $T_{c} \simeq 250 \mathrm{MeV}, \hat{q}_{\mathrm{LV}} \simeq 3.56 \mathrm{GeV}^{2} / \mathrm{fm}$, which is larger than $\hat{q}_{\text {conf. }}$. As temperature increases, $\hat{q}_{\mathrm{LV}}$ approaches

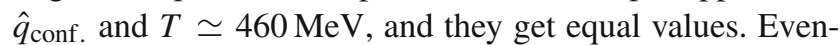
tually, for $T>460 \mathrm{MeV}$, the values of $\hat{q}_{\mathrm{LV}}$ become smaller than $\hat{q}_{\text {conf. }}$. From the numerical values of the jet quenching parameter, it should be mentioned that, in the range of $300<T<500$, we found $5.1<\hat{q}_{\mathrm{LV}}<14.2$, which is consistent with the results from RHIC, $\overline{\hat{q}} \simeq 5-15 \mathrm{GeV}^{2} / \mathrm{fm}$ $[81,82]$. We evaluated the jet quenching parameter as a function of temperature for different values of $\theta$ and $z$ in Fig. 4 . From this plot, one can observe that $\hat{q}_{\mathrm{LV}}$ becomes large as $\theta$ increases. For different values of $z$ and constant value of $\theta$, we plotted the jet quenching parameter as a function of temperature (Fig. 5). In this case, $\hat{q}_{\mathrm{LV}}$ increases as $\theta$ increases. Then we plotted the jet quenching parameter as a function of the hyperscaling exponent $\theta$ for different values of $T$ and fixed Lifshitz exponent $z$ in Fig. 6. From this figure, we found that $\hat{q}_{\mathrm{LV}}$ is an increasing function of $\theta \cdot \hat{q}_{\mathrm{LV}}$ as a function of $z$ is depicted in Fig. 7 for different values of $T$ and a constant value of $\theta$; like the previous case, it is an increasing function of $z$.

Finally, we added a constant B-field (electric field) to the background metric and estimated the dependency of the jet quenching parameter on the Hawking temperature as well as the electric field for $z=-8$ and $\theta=10$. From Fig. 8, one can observe that $\hat{q}_{\mathrm{LV}}$ rises as $T$ increases. From Fig. 9 it can be seen that, at constant temperature, $\hat{q}_{\mathrm{LV}}$ is an increasing function of $\mathcal{E}$.

Open Access This article is distributed under the terms of the Creative Commons Attribution License which permits any use, distribution, and reproduction in any medium, provided the original author(s) and the source are credited.

Funded by SCOAP 3 / License Version CC BY 4.0.

\section{References}

1. J.M. Maldacena, The large $\mathrm{N}$ limit of superconformal field theories and supergravity. Adv. Theor. Math. Phys. 2, 231 (1998). (Int. J. Theor. Phys. 38, 1113 (1999))

2. S.S. Gubser, I.R. Klebanov, A.M. Polyakov, Gauge theory correlators from non-critical string theory. Phys. Lett. B 428, 105 (1998)

3. O. Aharony, S.S. Gubser, J.M. Maldacena, H. Ooguri, Y. Oz, Large $\mathrm{N}$ field theories, string theory and gravity. Phys. Rept. 323, 183 (2000). arXiv:9905111 [hep-th]

4. E. Witten, Anti-de Sitter space and holography. Adv. Theor. Math. Phys. 2, 253-291 (1998). arXiv:9802150 [hep-th]

5. C.P. Herzog, A. Karch, P. Kovtun, C. Kozcaz, L.G. Yaffe, Energy loss of a heavy quark moving through $\mathrm{N}=4$ supersymmetric YangMills plasma. JHEP 0607, 013 (2006). hep-th/0605158

6. S.S. Gubser, S.S. Pufu, F.D. Rocha, A. Yarom, Energy loss in a strongly coupled thermal medium and the gauge-string duality. arXiv:0902.4041 [hep-th]

7. C.P. Herzog, Energy loss of heavy quarks from asymptotically AdS geometries. JHEP 09032 (2006). arXiv:0605191 [hep-th]

8. S.S. Gubser, Drag force in AdS/CFT. Phys. Rev. D 74, 126005 (2006) 
9. J.F. Vazquez-Poritz, Drag force at finite $t$ Hooft coupling from AdS/CFT. arXiv:0803.2890 [hep-th]

10. J. Sadeghi, M.R. Setare, B. Pourhassan, Drag force with different charges in STU background and AdS/CFT. J. Phys. G: Nucl. Part. Phys. 36, 115005 (2009)

11. J. Sadeghi, B. Pourhassan, Energy loss and jet quenching parameter in a thermal non-relativistic, non-commutative Yang-Mills plasma. Acta Physica Polonica B 43, 1825-1841 (2012)

12. M. Ali-Akbari, U. Gursoy, Rotating strings and energy loss in non-conformal holography. JHEP 01, 105 (2012). arXiv: 1110.5881 [hep-th]

13. E. Caceres, A. Guijosa, Drag force in charged $\mathrm{N}=4 \mathrm{SYM}$ plasma. JHEP 11, 077 (2006)

14. K. Bitaghsir Fadafana, H. Soltanpanahi, Energy loss in a strongly coupled anisotropic plasma. JHEP 01, 085 (2012). arXiv:1206.2271v4 [hep-th]

15. J. Sadeghi, M.R. Setare, B. Pourhassan, S. Hashmatian, Drag force of moving quark in STU background. Eur. Phys. J. C 61, 527 (2009). arXiv:0901.0217 [hep-th]

16. J. Sadeghi, B. Pourhassan, Drag force of moving quark at the $\mathrm{N}=$ 2 supergravity. JHEP 12, 026 (2008). arXiv:0809.2668 [hep-th]

17. B. Pourhassan, J. Sadeghi, STU/QCD correspondence. Can. J. Phys. 91, 995-1019 (2013). arXiv:1205.4254 [hep-th]

18. M. Chernicoff, J.A. Garcia, A. Guijosa, The energy of a moving quark-antiquark pair in an N = 4 SYM plasma. JHEP 09, 068 (2006)

19. T. Matsuo, D. Tomino, W.Y. Wen, Drag force in SYM plasma with B field from AdS/CFT. JHEP 10, 055 (2006)

20. M. Ali-Akbari, K.B. Fadafan, Rotating mesons in the presence of higher derivative corrections from gauge-string duality. Nucl. Phys. B 835, 221-237 (2010). arXiv:0908.3921v1 [hep-th]

21. J. Sadeghi, S. Heshmatian, Screening length of rotating heavy meson from AdS/CFT. Int J Theor Phys 49, 1811-1822 (2010). arXiv:0812.4816v3 [hep-th]

22. J. Sadeghi, M.R. Pahlavani, R. Morad, S. Heshmatian, Baryon binding energy in the Sakai-Sugimoto model. Int. J. Theor. Phys. 50(2), 488-496 (2011). arXiv:0910.3542v2 [hep-th]

23. J. Sadeghi, S. Heshmatian, Decay widths of large-spin mesons from the non-critical string/gauge duality. Phys. Rev. D 84, 126010 (2011). arXiv:1105.6273 [hep-th]

24. J. Sadeghi, B. Pourhassan, S. Heshmatian, Application of AdS/CFT in quark-gluon plasma. Adv. High Energy Phys. 2013, 759804 (2013)

25. R.-G. Cai, S. Chakrabortty, S. He, L. Li, Some aspects of QGP phase in a hQCD model. JHEP 02, 068 (2013). arXiv:1209.4512 [hep-th]

26. U. Gursoy, E. Kiritsis, Exploring improved holographic theories for QCD: Part I. JHEP 0802, 032 (2008). arXiv:0707.1324 [hep-th]

27. U. Gursoy, E. Kiritsis, F. Nitti, Exploring improved holographic theories for QCD: Part II. JHEP 0802, 019 (2008). arXiv:0707.1349 [hep-th]

28. U. Gursoy, E. Kiritsis, L. Mazzanti, F. Nitti, Holography and thermodynamics of 5D dilaton-gravity. JHEP 0905, 033 (2009). arXiv:0812.0792 [hep-th]

29. U. Gursoy, E. Kiritsis, L. Mazzanti, F. Nitti, Langevin diffusion of heavy quarks in non-conformal holographic backgrounds. JHEP 1012, 088 (2010). arXiv:1006.3261 [hep-th]

30. H. Singh, Lifshitz/Schrodinger Dp-branes and dynamical exponents. JHEP 1207, 082 (2012). arXiv: 1202.6533 [hep-th]

31. K. Narayan, On Lifshitz scaling and hyperscaling violation in string theory. Phys. Rev. D 85, 106006 (2012). arXiv:1202.5935 [hep-th]

32. K. Balasubramanian, K. Narayan, Lifshitz spacetimes from AdS null and cosmological solutions. JHEP 1008, 014 (2010). arXiv:1005.3291 [hep-th]

33. P. Dey, S. Roy, Intersecting D-branes and Lifshitz-like space-time. Phys. Rev. D 86, 066009 (2012). arXiv:1204.4858 [hep-th]
34. P. Dey, S. Roy, Lifshitz-like space-time from intersecting branes in string/M theory. JHEP 1206, 129 (2012). arXiv:1203.5381 [hep-th]

35. T. Azeyanagi, W. Li, T. Takayanagi, On string theory duals of Lifshitz-like fixed points. JHEP 0906, 084 (2009). arXiv:0905.0688 [hep-th]

36. S. Kachru, X. Liu, M. Mulligan, Gravity duals of Lifshitz-like fixed points, Phys. Rev. D 78, 106005 (2008). arXiv:0808.1725 [hep-th]

37. L. Huijse, S. Sachdev, B. Swingle, Hidden Fermi surfaces in compressible states of gauge-gravity duality. Phys. Rev. B 85, 035121 (2012). arXiv:1112.0573 [condmat. str-el]

38. E. Shaghoulian, Holographic entanglement entropy and fermi surfaces. JHEP 1205, 065 (2012). arXiv:1112.2702 [hep-th]

39. B. Gouteraux, E. Kiritsis, Generalized holographic quantum criticality at finite density, JHEP 1112, 036 (2011). arXiv:1107.2116 [hep-th]

40. C. Charmousis, B. Gouteraux, B.S. Kim, E. Kiritsis, R. Meyer, Effective holographic theories for low-temperature condensed matter systems. JHEP 1011, 151 (2010) arXiv:1005.4690 [hep-th]

41. S.A. Hartnoll, E. Shaghoulian, Spectral weight in holographic scaling geometries. JHEP 1207, 078 (2012). arXiv:1203.4236 [hep-th]

42. X. Dong, S. Harrison, S. Kachru, G. Torroba, H. Wang, Aspects of holography for theories with hyperscaling violation. JHEP 1206, 041 (2012). arXiv:1201.1905 [hep-th]

43. M. Edalati, J.F. Pedraza, Aspects of current correlators in holographic theories with hyperscaling violation. Phys. Rev. D 88, 086004 (2013). arXiv:1307.0808v2 [hep-th]

44. M. Edalati, J.F. Pedraza, W.T. Garcia, Quantum fluctuations in holographic theories with hyperscaling violation. Phys. Rev. D 87, 046001 (2013). arXiv:1210.6993v3 [hep-th]

45. B. Gouteraux, J. Smolic, M. Smolic, K. Skenderis, M. Taylor, Holography for Einstein-Maxwell-dilaton theories from generalized dimensional reduction. JHEP 1201, 089 (2012). arXiv: 1110.2320 [hep-th]

46. B.S. Kim, Schrodinger holography with and without hyperscaling violation. JHEP 1206, 116 (2012). arXiv:1202.6062 [hep-th]

47. E. Perlmutter, Domain wall holography for finite temperature scaling solutions. JHEP 1102, 013 (2011). arXiv:1006.2124 [hep-th]

48. E. Perlmutter, Hyperscaling violation from supergravity. JHEP 1206, 165 (2012). arXiv:1205.0242v2 [hep-th]

49. P. Bueno et al., Lifshitz-like solutions with hyperscaling violation in ungauged supergravity. JHEP 1301, 189 (2013). arXiv:1209.4047 [hep-th]

50. P. Bueno et al. On hvLif-like solutions in gauged supergravity. Eur. Phys. J. C 74, 2684 (2014). arXiv:1212.4826 [hep-th]

51. J. Gath, J. Hartong, R. Monteiro, N.A. Obers, Holographic models for theories with hyperscaling violation. JHEP 1304, 159 (2013). arXiv: 1212.3263 [hep-th]

52. M. Taylor, Non-relativistic holography. arXiv:0812.0530 [hep-th]

53. M. Alishahiha, H. Yavartanoo, On holography with hyperscaling violation. JHEP 1211, 034 (2012). arXiv:1208.6197 [hep-th]

54. M. Alishahiha, E.O Colgain, H. Yavartanoo, Charged black branes with hyperscaling violating factor. JHEP 11, 137 (2012). arXiv:1209.3946 [hep-th]

55. J. Sadeghi, B. Pourhassan, A. Asadi, Application of hyperscaling violation in QCD. Can. J. Phys. 92, 280-283 (2014)

56. E. Kiritsis, Lorentz violation. Gravity, dissipation and holography. JHEP 1301, 030 (2013). arXiv:1207.2325 [hep-th]

57. J. Sadeghi, B. Pourhasan, F. Pourasadollah, Schrodinger black holes with hyperscaling violation. Phys. Lett. B 720, 244 (2013). arXiv:1209.1874 [hep-th]

58. J.D. Bjorken, Energy Loss Of Energetic Partons In Quark-Gluon Plasma: Possible Extinction Of High P(T) Jets In Hadron-Hadron Collisions, FERMILAB-PUB-82-059-THY

59. A. Kovner, U.A. Wiedemann, in Gluon radiation and parton energy loss, ed. by R.C. Hwa et al., Quark gluon plasma* pp. 192-248. arXiv:0304151 [hep-ph] 
60. S.S. Adler et al. [PHENIX Collaboration], Nuclear modification of electron spectra and implications for heavy quark energy loss in $\mathrm{Au}+\mathrm{Au}$ collisions at $\mathrm{s}(\mathrm{NN}) * *(1 / 2)-200-\mathrm{GeV}$. Phys. Rev. Lett. 96, 032301 (2006). arXiv:0510047 [nucl-ex]

61. J. Bielcik [STAR Collaboration], Centrality dependence of heavy flavor production from single electron measurement in $\mathrm{s}(\mathrm{NN}) * *(1 / 2)=200-\mathrm{GeV} \mathrm{Au}+$ Au collisions. Nucl. Phys. A 774, 697 (2006). arXiv:0511005 [nucl-ex]

62. R. Baier, Y.L. Dokshitzer, A.H. Mueller, S. Peigne, D. Schiff, Radiative energy loss and $\mathrm{p}(\mathrm{T})$-broadening of high energy partons in nuclei. Nucl. Phys. B 484, 265 (1997)

63. F. D'Eramo, H. Liu, K. Rajagopal, Transverse momentum broadening and the jet quenching parameter, redux. Phys. Rev. D 84, 065015 (2011). arXiv:1006.1367 [hep-ph]

64. A. Adare et al. [PHENIX Collaboration], Energy loss and flow of heavy quarks in $\mathrm{Au}+\mathrm{Au}$ collisions at $\mathrm{psNN}=200 \mathrm{GeV}$. Phys. Rev. Lett. 98172301 (2007). arXiv:0611018 [hep-th]

65. F. Bigazzi, A.L. Cotrone, J. Mas, A. Paredes, A.V. Ramallo, J. Tarrio, D3-D7 Quark-Gluon Plasmas. JHEP 0911, 117 (2009). arXiv:0909.2865 [hep-th]

66. U. Gursoy, E. Kiritsis, G. Michalogiorgakis, F. Nitti, Thermal transport and drag force in improved holographic QCD. JHEP 0912, 056 (2009). arXiv:0906.1890v4 [hep-ph]

67. K.B. Fadafan, Medium effect and finite tHooft coupling correction on drag force and jet quenching parameter. Eur. Phys. J. C 68, 505-511 (2010). arXiv:0809.1336 [hep-th]

68. D. Giataganas, Probing strongly coupled anisotropic plasma. JHEP 1207, 031 (2012). arXiv:1202.4436v2 [hep-th]

69. E. Caceres, A. Guijosa, On drag forces and jet quenching in strongly coupled plasmas. JHEP 12068 (2006)

70. H. Liu, K. Rajagopal, U.A. Wiedemann, Calculating the jet quenching parameter from AdS/CFT. Phys. Rev. Lett. 97, 182301 (2006). arXiv:0605178 [hep-ph]
71. H. Liu, K. Rajagopal, U.A. Wiedemann, Wilson loops in heavy ion collisions and their calculation in AdS/CFT. JHEP 0703, 066 (2007). arXiv:0612168 [hep-ph]

72. A. Buchel, On jet quenching parameters in strongly coupled non-conformal gauge theories. Phys. Rev. D 74, 046006 (2006). arXiv:0605178 [hep-th]

73. H. Liu, K. Rajagopal, Y. Shi, Robustness and infrared sensitivity of various observables in the application of AdS/CFT to heavy ion collisions. JHEP 0808, 048 (2008). arXiv:0803.3214 [hep-ph]

74. J.F. Vazquez-Poritz, Enhancing the jet quenching parameter from marginal deformations. arXiv:0605296 [hep-th]

75. F.L. Lin, T. Matsuo, Jet quenching parameter in medium with chemical potential from AdS/CFT. Phys. Lett. B 64145 (2006)

76. E. Nakano, S. Teraguchi, W.Y. Wen, Drag force, jet quenching and AdS/QCD. Phys. Rev. D 75085016 (2007)

77. S.D. Avramis, K. Sfetsos, Supergravity and the jet quenching parameter in the presence of R-charge densities. JHEP 01065 (2007)

78. N. Armesto, J.D. Edelstein, J. Mas, Jet quenching at finite t Hooft coupling and chemical potential from AdS/CFT. J. High Energy Phys. JHEP 09039 (2006)

79. K. Bitaghsir Fadafan, B. Pourhassan, J. Sadeghi, Calculating the jet-quenching parameter in STU background. Eur. Phys. J. C 71, 1785 (2011)

80. S.S. Gubser, Curvature singularities: the good, the bad, and the naked. Adv. Theor. Math. Phys. 4, 679-745 (2000). arXiv:0002160 [hep-th]

81. K.J. Eskola et al., Nucl. Phys. A 747, 511 (2005)

82. A. Dainese, C. Loizides, G. Paic, Eur. Phys. J. C 38, 461 (2005) 